\title{
Personalized Pain Medicine: Turning Theory into Policy
}

\author{
Fatemeh Ramezani ${ }^{1}$, Bijan Riazi Farzad ${ }^{2}$ and Atousa Janzadeh (ii ${ }^{3,{ }^{*}}$ \\ ${ }^{1}$ Iran University of Medical Sciences, Tehran, Iran \\ ${ }^{2}$ UCL Institute of Education, London, UK \\ ${ }^{3}$ Occupational Medicine Research Center (OMRC), Iran University of Medical Sciences (IUMS), Tehran, Iran \\ "Corresponding author: Occupational Medicine Research Center (OMRC), Iran University of Medical Sciences (IUMS), Tehran, Iran. Email: atousajanzadeh@gmail.com
}

Received 2021 May 22; Accepted 2021 September 04.

\begin{abstract}
On the one hand, patients, therapists, policy-makers, business people, and health organization managers need to approach medicine from different perspectives, each perspective being appropriate for each role. On the other hand, a lack of appreciation of the perspectives of the other vested interests diminishes their effectiveness in accomplishing what their respective roles demand. In this article, we explain the main difference between these perspectives along a spectrum ranging from highly individualist to highly collectivist views of medicine. We aim to show the gap between personal (individualistic) and social (collectivist) medicine models. We then present possible ways to close this gap. We argue that these differences need to be reconciled, at least to some extent, if medicine is to evolve along with other disciplines, such as engineering, to create and implement personalized solutions to patients' pains. We conclude by proposing a framework through which patients, practitioners, health organizations, business people, and policy-makers can develop enough of a mutual understanding of each other's perspectives, problems, and solution orientations to be able to work relatively harmoniously toward the common goal of creating bespoke solutions to individuals' pains.
\end{abstract}

Keywords: Personalized Medicine, Pain, Genetics, Epigenetics, Database

\section{Background}

This paper calls for establishing an interdisciplinary framework for the universal implementation of personalized pain medicine (PPM). Let us begin by examining this concept within a broader context.

Medicine is personal. Moreover, nothing is more personal than being free from pain. Medicine only becomes impersonal when it becomes a matter of business or governance. Therefore, in an ideal society, the phrase 'personalized medicine' would be considered tautologous.

Pain is the archenemy of happiness because it disrupts our jobs, self-confidence, relationships with our families and friends, and lifestyle in general. Every year, approximately 560 to 635 billion dollars are spent in the United States to treat pain. These figures are "larger than the cost of the nation's priority health conditions" (1). In one study, it was found that $44 \%$ of the interviewed subjects reported having experienced pain on the day of the interview. Ten percent of these were chronic cases who visited their physicians 11 times each year on average. The researchers concluded that "in the general population in Israel, widespread pain was common, and its prevalence was comparable to reports from USA, UK, and Canada." Meanwhile, other studies have reported that, in the United
States, around $20 \%$ of the adult population suffer from chronic pain and, despite the fact that around half of them receive analgesic treatment, $64 \%$ continue to experience pain (2). Clearly, in addition to taking its personal toll, pain is a huge burden on the economy.

The problem has been that nature thrives on diversity. One size cannot fit all because everyone is not the same size. Our anatomy and physiology are as diverse as our fingerprints. It is this diversity that makes much of criminology possible. On the other hand, business models and governance systems are mainly based on Taylorism; expansion through standardization, repetition, replication, and given half a chance, ultimately, cloning.

Things are changing, however. Since the discovery of the polymerase chain reaction in the late 20th century and the advent of the Human Genome Project, medicine has been able to move toward genuinely celebrating our diversity. This has become possible because we are now able to easily map our differences at the genetic level. This is, of course, a technological breakthrough, and established models of business and governance have traditionally been slow to adapt to technology.

This article is about how scientists and health professionals may help businesses and, more particularly, cur- 
rently less adaptive systems of governance to move forward with the times. Personalization, including personalization of medicine, is no longer for the elite, and businesses and systems of governance that fail to adapt will suffer the same fate as Darwin's misfits.

Our aim here is to demonstrate that there is a gap between the personal (individualist) and social (collectivist) models (perspectives) of medicine. We then propose possible ways to bridge this gap. To this end, a framework needs to be created through which the collectivist (business and political) and individualist (patient and therapist) perspectives of medicine can come together. Such common frames of reference are a necessary first step to establishing a productive dialogue between the different players in this arena. This means that patients, scientists, and therapists need to appreciate traditional constraints that enchain business and politics. In contrast, business and political communities need to appreciate that much of what appear to be constraints are illusory social constructs handed down to us by our forefathers.

The second step in our quest for helping create the requisite business and political will to prioritize the personalization of medicine in general, and pain management in particular, is to use these common frames of reference to show that a personalized model of medicine can fit (or at least not to conflict with) the collectivist models of business and governance.

Appreciating that such common understandings are useless and a waste of time, unless they can be empowered through positive action, we next intend to facilitate the process through which business and governance can implement personalized medicine, thereby helping turn theory into policy. We aim to do this by proposing various models that can act as scaffolds to guide and support the practical implementation of personalized medicine whereby members of the business and political community can see themselves as part of a team that includes scientists, medical practitioners, and patients. Ultimately, our goal is to do our part in breaking down barriers between policy-makers and those affected by such policies, which, ironically, include policy-makers themselves.

The first leg of this journey is what we call personalized pain medicine (PPM) standardization. However, let us begin by putting this into a much broader context; our vision.

\subsection{The Urgent Need for Personalization of Pain Medicine}

Focusing on the treatment of pain, studies have shown that the quest to develop a panaceum for relieving pain has consistently led to costly disappointment. To quote Allen Roses, worldwide vice-president of genetics at GlaxoSmithKline, "the vast majority of drugs - more than 90 percent - only work in 30 or 50 percent of the people" (3).
This statement becomes even more alarming when we consider that many studies have indicated that these figures are close to the effect size of the placebo response. In other words, similar or, in some cases, better results can be obtained through the placebo effect. For example, published in 2016, a meta-analysis of the effectiveness of various treatments on relieving the pain of osteoarthritis concluded that "contextual effects explained $85 \%$ of the improvement with topical NSAIDs," and that "on average, the contextual effect contributed $75 \%$ to the overall treatment effect for the included treatments for pain in osteoarthritis" $(4,5)$. Similarly, in their 2018 paper entitled, A Comprehensive Review of Non-Steroidal Anti-inflammatory Drug Use in the Elderly, the researchers pointed out that in 2015, Jankowski et al. conducted a randomized controlled trial of 189 elderly subjects between 60 and 75 years of age (6). They determined the effects of ibuprofen use and bone mineral density adaptations after 36 weeks of exercise. They found no difference between treatment groups and the placebo (7).

As we mentioned earlier, part of the problem is that we are more distinctive on the inside (physiologically) than a cursory look at our anatomy may suggest. Since the latter parts of the twentieth century, there has been a wave of interest in our genetic diversity, and research has demonstrated that our bodies' responses to various treatments vary depending on our genetic makeup.

\subsection{OurVision}

Let us invite you to join us in a thought experiment. Imagine yourself moving to a time in the, hopefully, not so distant future. You are speaking to a close friend or family member when he or she suddenly complains of severe pain. You call for an ambulance. The ambulance is on its way. The person at the other end of the line only asks for your companion's 'PPM code' and whether there has been any recent trauma.

On arrival at the hospital, doctors have already examined your companion's medical records and determined:

(a) The kind of injury your companion is most susceptible to under normal circumstances,

(b) The treatment regimen that is unlikely to be effective,

(c) Priority areas for direct medical examination in the absence of recent trauma,

(d) The kind of medical procedures your companion is most likely to tolerate well.

The medical team identifies the etiology of your companion's pain accurately and confidently within minutes of their arrival. The person is given a personalized treatment regimen that effectively alleviates their pain and suppresses the condition responsible for it. 
Reflecting on this experience, you marvel at how quickly the morbidity rate has decreased and how much healthier the world has become. There are now noticeably fewer hospitals than there were at the beginning of the decade of the 2020s. The new generation of physicians read about palliative care in hospitals of your generation and wonder how society ever survived before 'PPM standardization'. You smile as you remember your reaction when your parents told you about when there was no internet, and you stood there wondering how people survived in such backward times.

This is the promise of PPM.

\subsection{How is it Possible?}

At the heart of PPM is a database. At its simplest, a database is a two-column table linking one variable to another. The internet's Domain Name System, a multimillion-dollar industry, is such a database linking domain names to IP addresses. This example demonstrates how a straightforward solution can be useful, effective, and very lucrative.

The PPM database that we are proposing will need to be somewhat more elaborate than that. However, it will essentially include rows of data, with each row (record) consisting of various pieces of information (fields) about a particular individual. This information will be pooled together from various sources. Similar work has been successfully carried out in other disciplines, such as education (8). This precedence is a valuable demonstration of what can be achieved. In the study, a database was created containing over 23,500 records (students), with each record consisting of over six hundred pieces of information about each student's social, academic, and psychological background. This data was gathered through national databases, school records, questionnaires, interviews with the students and their teachers, and direct (ethnographic) observation.

We similarly need to gather information from various sources for the PPM database, including national databases, hospital records, GP records, laboratory reports, family records, and, of course, patients themselves. These records will then provide us with a genetic profile and an epigenetic profile of each individual. We discuss the details of genetic and epigenetic profiling in a separate paper.

At this stage, we want to point out that to coordinate our efforts to create a functional and productive database, we need the cooperation of many stakeholders, including patients, healthcare professionals, healthcare administrative centers, medical laboratories, medical research centers, and their researchers, the pharmaceutical industry, private businesses, governments, and grant-awarding bodies.

\subsection{The Fundamentals of a PPM Roadmap}

1.4.1. One Objective, One Problem, and One Course Correction

We have a dream, but we call it an objective since this is not a political rally. It sounds simple enough: we want to minimize world pain. Zooming out of our utopian frame of mind, and looking at the world from the point of view of the health sector and the medical industry at large, if we reduce pain to levels that are below a certain threshold, current business models will no longer be viable and, as any businessperson or capitalist can tell you, this is like trying to bite the hand that feeds us. However, if you had to choose between living in a world where pain was incidental and keeping your current job, which would you choose? From a more pragmatic point of view, we can regard this objective as a problem to be solved: How can we make PPM cost-effective?

Taking some inspiration from the experiences and trends in the automotive industry, we believe that PPM can become cost-effective when we strike a balance between our differences (what makes us individuals) and what we have in common (what makes us members of the same species-or phylum even).

When we think of societies and what holds them together, we tend to focus on what individuals have in common in these societies. This is similar to how when we use animal models, we assume that the physiology of a mouse, for example, is sufficiently similar to the physiology of a human to make it a valid model. Meanwhile, we do not consider the mouse as being sufficiently similar to us to warrant bestowing the same fundamental human rights on it.

The same duality (one could call it hypocrisy) has led us down a misleading path in our attempts to design effective treatments for pain. That is, we have been trying to design pain medication on the premise that we all share the same physiology. Not surprisingly (and, of course, in hindsight), this has shown itself to have severe limitations. Therefore, the one course correction that we intend to highlight in this article is that one size does not fit all.

\subsubsection{A Spectrum Along with Two Perspectives}

We have surmised that to move toward our objective, we need to consolidate two seemingly opposing perspectives: individualism and collectivism. Each of these terms can be interpreted differently in different contexts depending on our mindset at the time.

Depending on our perspectives and attitudes toward the concepts of 'individualism' and 'collectivism', each idea evokes words imbued with value judgments that lie on a desirable-detestable spectrum.

From the perspective of management and governance, individualism threatens the fluent fabric of the organization or society. From the perspective of a person suffering 
from debilitating pain, waiting in line is a sign that the society (management / leadership) does not care about its citizens.

It is clear, therefore, that the difference is not in the terms we use but in our attitudes toward 'the Other' (also referred to as 'Otherness' and the Constitutive Other' (911). At one extreme, the more detached we become from the sufferer, the more we move toward 'psychosis', and at the other extreme, the more attached we become to the sufferer, the more 'neurotic' we become in our approach to treatment. With regards to the latter, according to the American Medical Association's Code of Ethics 1.2.1, "treating oneself or a member of one's own family poses several challenges for physicians, including concerns about professional objectivity, patient autonomy, and informed consent." In short, when attempting to reconcile the views of patients and their practitioners on one side and those of businesses and governance on the other, we need to strike a delicate balance because moving to either extreme would lead to pathological outcomes on a social scale.

In order to create effective PPM protocols, we need to consider interactions between three variables; genes, the individual's environment, and social policies (12-16). Since detailed consideration of these variables is beyond the scope of this paper, we focus on these interactions in a separate paper.

\section{Seeking Common Ground}

To consider any individual more worthy of being free from pain than any other is to renounce our membership of the human race.

Many of our social and psychological problems, including, paradoxically, memberships of antisocial gangs and groups, have been shown to stem from our need to belong (17). If we do not belong to a constructive society, we would rather belong to a destructive one than not be part of anything.

In certain professions, such as business and governance, it is necessary to take a step back and look at the whole. Otherwise, managers would not be able to 'see the wood for the trees'. However, the reverse can also be argued; one can become so caught up in the wood that one would fail to appreciate its individual trees. Studies have shown that despite popular lore, whether or not managers and top leaders feel lonely is not a matter of their rank or position but how they see their connection with others. It depends on the extent to which, having seen the wood, they can return to the trees. In other words, a lonely leader is a divisive leader, while a happy leader sees themselves as a part of those whom they are leading. Therefore, a happy leader shares the pain of those whom they are leading. They lead from the front.

We have already alluded to what we believe to be the first common ground between the collectivist and individualist perspectives: each human being has intrinsic value. If this value does not manifest itself at any particular moment, it is because the current environmental conditions are not conducive for its manifestation.

The second step in our quest for helping create the requisite business and political will to prioritize the personalization of medicine in general, and pain management in particular, is to use common frames of reference to show that a personalized model of medicine can fit (or at least not conflict with) the collectivist models of business and governance.

\section{The Roadmap}

In order to help policy-makers implement the principles outlined here, and for those affected by these policies to embrace them, suitable frameworks are needed. These frameworks are called blueprints in the construction business. They are referred to as business plans within the business community and strategies in governance. Also, scientists call these frameworks models, and cooks call them recipes.

Here, we have settled on the term 'roadmap'. The following schema (Figure 1) presents the outline of a roadmap as we envisage it at present.

\subsection{Ground 0: This Paper}

In this paper, we hope to have planted a seed, an opportunity that, if nurtured, can create something that will be greater than the sum of its parts. We believe that our growing knowledge of the complex range of phenomena that we simplistically call 'pain', coupled with our growing appreciation of the inadequacies of our current systems of pain management and treatment, presents us with an unprecedented and compelling opportunity to catapult medical practice, biomedical research, and global health services into a new era. This era encompasses global personalized medicine in general and PPM in particular.

We believe that the critical question here is not "how realizable is this opportunity?" Instead, it is, "can we create enough enthusiasm to do what is already being done on a small scale on a global scale?" Some of our colleagues 'in the business' have told us that even the idea of a national program for PPM would be too expensive to implement, let alone a global one. We take heart in the well-known story of Henry Ford's resolution and the birth of the V8 engine that transformed the automotive industry, making a motorcar affordable for the masses. Henry Ford had a team of 


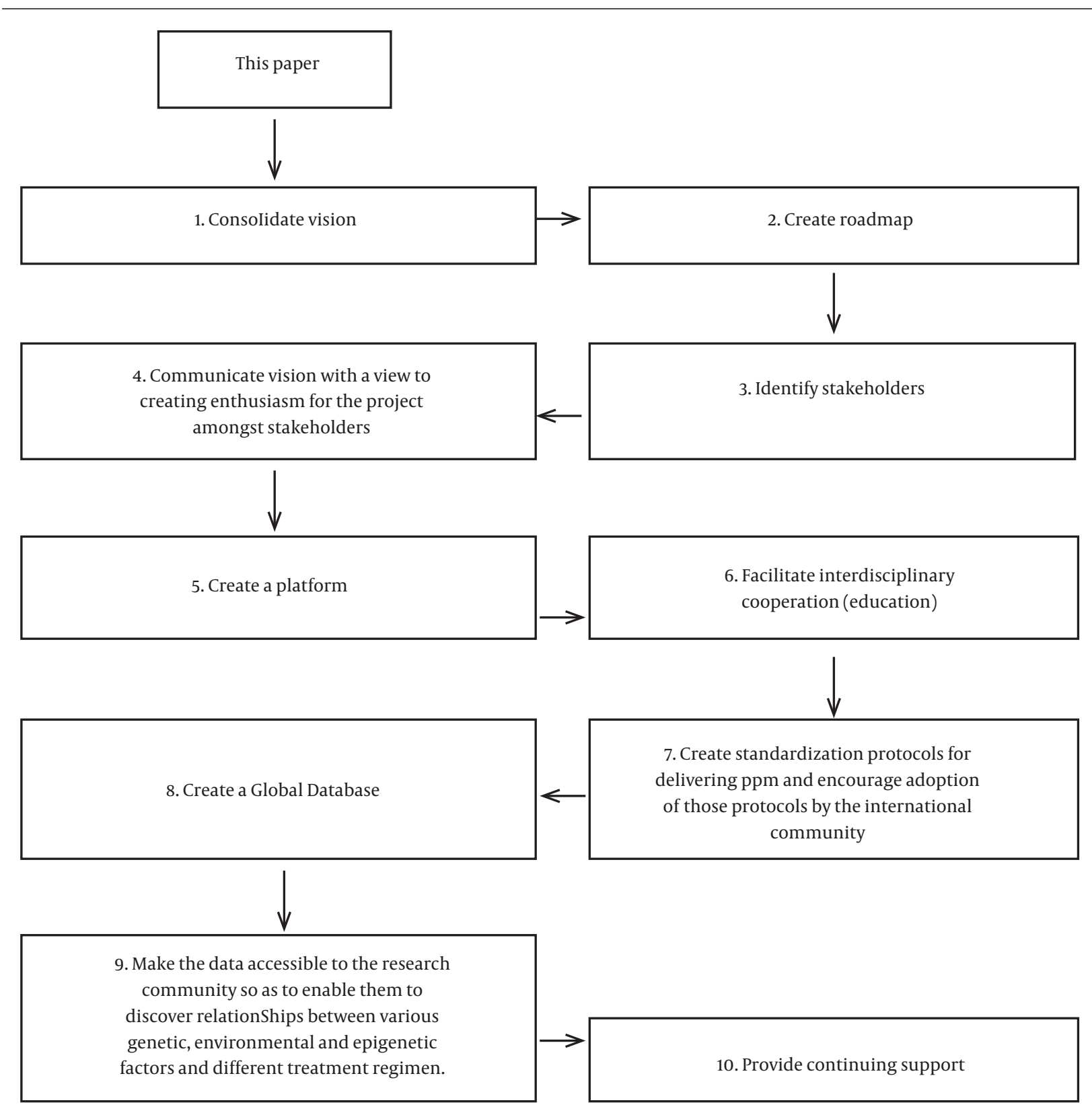

Figure 1. A proposed roadmap for the creation of a global center for PPM

engineers that he burdened with the task of making what they said was 'impossible' possible. Therefore, in one sense, this article is a call for anyone who is enthused by the idea of making the 'impossible' possible, whether it be from within the medical community, the business community, or the political community, to rise to the challenge.

\subsection{Stage 1. Consolidate Vision}

We acknowledge that our proposal is ambitious and has many loose ends to be tied up before it can turn from a vision into a solid proposal. We needed to start somewhere and thus examined an article in the Lancet in the first step. Robert Townsend is the man who transformed Avis Renta-Car from a failing company into a soaring success. In his book, Up The Organization $(18,19)$, he says that two meetings are better than one, explaining that, "At the first [meeting], 
you pick up the convictions of the quick reactors, and at the second, you give equal time to the just-as-valuable convictions of those who should sleep on it." We pose this article as Townsend's 'first meeting' and look forward to hearing from those 'who should sleep on it'.

\subsection{Stage 2. Create a Roadmap}

Currently, there are doctors, nurses, business people, biotechnologists, lobbyists, and national health departments in many countries and many others who, through their disciplines, are making contributions to alleviate pain and suffering all around the globe. Imagine, if you would, a metaphorical bus. The destination of the bus is a global health research and an action center from where solutions to everyone's pain problems emanate. The bus needs to pick a route that allows it to pick up activists and stakeholders along the way and deliver them to the global center. At present, we have many potential participants in this project, but they need to be identified and engaged. In this day and age, creating a global center that is to be their destination is not difficult to establish since it can be, and may indeed need to be, virtual to begin with.

\subsection{Stages 3 and 4. Identify and Enthuse Stakeholders}

The process of writing this article and creating a website has opened our eyes to many untapped opportunities that need to be explored and realized. Stakeholders need to be identified (or, hopefully, self-identify) to join the project. We also need to communicate this vision to stakeholders to create enthusiasm for the project. We hope that readers of this article will also help disseminate this vision to maximize participation.

\subsection{Stage 5. Create a Platform}

At present, we have an idea, an article, a website, and a group of dedicated professionals with a vision and the will to realize the vision.

We intend to expand this to create a platform for stakeholders to rally around and transform it into a central bureau for the standardization of PPM, which will continue to bring together stakeholders and lead to the harmonization of the process.

\subsection{Stage 6. Facilitate Interdisciplinary Cooperation}

The following is an outline of a syllabus that we have conceived to further the cause of global PPM. It is a draught outline aiming to bring together various stakeholders to establish a global network to internationalize personal pain medicine (Figure 2).

This outline needs to be consolidated with the help of stakeholders, and the details of the content need to be compiled and formalized.
3.7. Stage 7. Create Standardization Protocols for Delivering PPM and Encourage Adoption of Those Protocols by the International Community

Even with genetic profiling, many different protocols are currently in use in different laboratories around the world. The harmonization process discussed here will involve the creation of standardized protocols. Identifying best practices and standardizing these procedures will help make the data shareable, making results compatible, enriching the global database, and reducing measurement errors. We then need to encourage the business and research community to adopt these protocols.

\subsection{Stage 8. Create a Global Database}

Various countries are creating genetic profile databases, especially concerning criminology.

For the purpose of developing a PPM database, existing systems have several shortcomings. In the first instance, there is a big difference between using genetics to identify people and addressing individuals' physiological strengths and weaknesses. Secondly, there is a lack of global standards for such protocols. Furthermore, as we have pointed out here, genetic information by itself is not enough where medicine is concerned since the environment and consequent epigenetic changes play an essential role in our response to treatment. Finally, our genetics, epigenetics, and medical history need to be matched against a database of treatment protocols, including medication.

\subsection{Stage 9. Make the Data Accessible to the Research Commu-} nity

Without data, there can be no science, no scientific discoveries, and no scientific progress. The quality and quantity of data are the primary determinants of the reliability and validity of research findings. We believe that a global database of the type suggested in Stage 8 can transform the medical sciences in the same way that the availability of physical data tables transformed the physical sciences.

\subsection{Stage 10. Provide Continuing Support}

With the help of our colleagues across the globe, we hope that a 'PPM development and standardization support centre' can be established to provide guidelines to researchers to help them conduct their research in a manner that will be compatible with the global database. This institution can also provide continuing support for centers that adopt the international PPM protocols. 
1. Public understanding of the origins of pain

2. Perspectives on PPM: pieces of a puzzle or links in a chain?

3. Transitioning from a social view to a personal view of medical science and vice versa.

4. Basic principles of personalized medicine; theory and practice.

5. Economic perspectives on PPM, including the economics of establishing PPM infrastructure (short-term costs versus long-term benefits)

6. The role of governance in PPM

7. The role of business in PPM

8. Modelling best practice and innovating new approaches to the implementation of PPM.

Figure 2. Various stakeholders to establish a global network for internationalizing personal pain medicine

\section{Conclusions}

The idea of central tendency, known to statisticians and other probability theorists, is known by different names in different disciplines: chemists see it in chemical equilibria; it is called homeostasis in physiology; it is known as maintaining the status quo in sociology; and it manifests as our psychological resistance to change in psychology.

Chemists and biochemists know that breaking barriers to change is achieved much more easily through a catalyst. In marketing, similar resistance to change is experienced as customers' reluctance to purchase goods or services. In such cases, the catalyst is what 'sweetens the deal'. Within the realm of politics, catalysts include the media and lobbyists.

This article implies that the catalyst that can bring the two sides (collectivist vs. personalized) of medicine together is education.

Education is a two-way process through which a learner becomes a part of what is learned and vice versa.

Applying this principle to our current discussion, for personalized medicine to establish itself within the realm of collectivist understandings such as governance and business, those practicing personalized medicine, including patients, scientists, technicians, and technologists, will also need to move toward the collectivist understanding of the world. Individualists can move toward an understanding of the collectivist perspective by asking the question, "how can we help as many people as possible?"

Conversely, for the business and governance community to appreciate the individualist perspective, we can begin by asking, "how can personalization of pain medicine improve my effectiveness as a business and as a government?"

\section{Acknowledgments}

The authors would like to thank the Iran University of Medical Sciences, the UCL Institute of Education, and the International Consortium for Personalized Pain Medicine (ICPPM).

\section{Footnotes}

Authors' Contribution: Study concept and design: Dr. Atousa Janzdeh, Dr. Bijan Riazi Farzad; Drafting of the manuscript: Dr. Fatemeh Ramezani.

Conflict of Interests: There were no relevant financial interests or conflicts related to the manuscript.

Ethical Approval: IR.IUMS.REC.1399.1288.

Funding/Support: The Iran University of Medical Sciences (IUMS) supported this manuscript.

Informed Consent: We did not have human subjects.

\section{References}

1. Gaskin DJ, Richard P. The economic costs of pain in the United States. J Pain. 2012;13(8):715-24. doi: 10.1016/j.jpain.2012.03.009. [PubMed: 22607834].

2. Hardt J, Jacobsen C, Goldberg J, Nickel R, Buchwald D. Prevalence of chronic pain in a representative sample in the United States. Pain Med. 2008;9(7):803-12. doi: 10.1111/j.1526-4637.2008.00425.x. [PubMed: 18346058].

3. Connor S. Glaxo chief: Our drugs do not work on most patients. The Independent. 2003;8:1-2.

4. Zou K, Wong J, Abdullah N, Chen X, Smith T, Doherty M, et al. Examination of overall treatment effect and the proportion attributable to contextual effect in osteoarthritis: meta-analysis of randomised controlled trials. Ann Rheum Dis. 2016;75(11):1964-70. doi: 10.1136/annrheumdis-2015-208387. [PubMed: 26882927]. [PubMed Central: PMC5099197]. 
5. Fu Y, Persson MS, Bhattacharya A, Goh SL, Stocks J, van Middelkoop M, et al. Identifying placebo responders and predictors of response in osteoarthritis: a protocol for individual patient data meta-analysis. Syst Rev. 2016;5(1):1-6. doi: 10.1186/s13643-016-0362-x. [PubMed: 27793184]. [PubMed Central: PMC5084436].

6. Jankowski CM, Shea K, Barry DW, Linnebur SA, Wolfe P, Kittelson J, et al. Timing of Ibuprofen Use and Musculoskeletal Adaptations to Exercise Training in Older Adults. Bone Rep. 2015;1:1-8. doi: 10.1016/j.bonr.2014.10.003. [PubMed: 25642444]. [PubMed Central: PMC4310009].

7. Wongrakpanich S, Wongrakpanich A, Melhado K, Rangaswami J. A Comprehensive Review of Non-Steroidal Anti-Inflammatory Drug Use in The Elderly. Aging Dis. 2018;9(1):143. doi: 10.14336/AD.2017.0306. [PubMed: 29392089]. [PubMed Central: PMC5772852].

8. Reiss M, Hoyles C, Mujtaba T, Riazi-Farzad B, Rodd M, Simon S, et al. Understanding Participation Rates in Post-16 Mathematics and Physics: Conceptualising and Operationalising the Upmap Project. Int J Sci Math Educ. 2011;9(2):273-302. doi:10.1007/s10763-011-9286-z.

9. Hawkesworth M. From Constitutive Outside to the Politics of Extinction: Critical Race Theory, Feminist Theory, and Political Theory. Polit Res Q. 2010;63(3):686-96. doi: 10.1177/1065912910367496.

10. Richter H. Beyond the 'other' as constitutive outside: The politics of immunity in Roberto Esposito and Niklas Luhmann. EurJ Political Theory. 2019;18(2):216-37. doi: 10.1177/1474885116658391.

11. Gunnarsson L. The Dominant and its Constitutive Other: Feminist Theorizations of Love, Power and Gendered Selves. J Crit Realism. 2016;15(1):1-20. doi: 10.1080/14767430.2015.1136194.

12. Kaye AD, Garcia AJ, Hall OM, Jeha GM, Cramer KD, Granier AL, et al. Update on the pharmacogenomics of pain management. Phar- mgenomics Pers Med. 2019;12:125. doi: 10.2147/PGPM.S179152. [PubMed: 31308726]. [PubMed Central: PMC6613192].

13. Mills SEE, Nicolson KP, Smith BH. Chronic pain: a review of its epidemiology and associated factors in population-based studies. $\mathrm{Br} J$ Anaesth. 2019;123(2):e273-83. doi: 10.1016/j.bja.2019.03.023. [PubMed: 31079836]. [PubMed Central: PMC6676152].

14. Kabore JL, Dassieu L, Roy E, Jutras-Aswad D, Bruneau J, Page MG, et al. Prevalence, Characteristics, and Management of Chronic Noncancer Pain Among People Who Use Drugs: A Cross-Sectional Study. Pain Med. 2020;21(11):3205-14. doi: 10.1093/pm/pnaa232. [PubMed: 32869088].

15. Gross J, Gordon DB. The Strengths and Weaknesses of Current US Policy to Address Pain. Am J Public Health. 2019;109(1):66-72. doi: 10.2105/AJPH.2018.304746. [PubMed: 30495994]. [PubMed Central: PMC6301412].

16. O'Brien T, Christrup LL, Drewes AM, Fallon MT, Kress HG, McQuay HJ, et al. European Pain Federation position paper on appropriate opioid use in chronic pain management. Eur J Pain. 2017;21(1):319. doi: 10.1002/ejp.970. [PubMed: 27991730]. [PubMed Central: PMC6680203].

17. Van Ngo H, Calhoun A, Worthington C, Pyrch T, Este D. The Unravelling of Identities and Belonging: Criminal Gang Involvement of Youth from Immigrant Families. J Int Migr Integr. 2015;18(1):63-84. doi: 10.1007/s12134-015-0466-5.

18. Grego P. Cornwall's Strangest Tales: Extraordinary but true stories. Portico; 2013.

19. Townsend RC, Bennis W. Up the organization: How to stop the corporation from stifling people and strangling profits. 144. John Wiley \& Sons; 2007. 